\title{
Les relations agriculture-élevage
}

Oléagineux, Corps Gras, Lipides. Volume 11, Numéro 4, 253-5, JUILLET-OCTOBRE 2004, AGRICULTURE / ÉLEVAGE : NORD-SUD

Auteur(s) : Philippe LHOSTE

\section{ARTICLE}

L'association de l'agriculture et de l'élevage (AAE) est une réalité diverse, complexe et controversée : panacée ou solution miracle pour les uns, discours incantatoire ou technique limitée pour les autres. II est vrai que les conditions de cette intégration sont très variables selon les situations agraires auxquelles on s'intéresse et que ces conditions varient également dans le temps.ll n'en reste pas moins que cette solution a profondément marqué l'évolution des agricultures du Monde, avec, certes, de forts décalages historiques [1-3]. Cette association que l'on peut aussi qualifier de " polyculture-élevage " est en effet, sans doute, encore de nos jours, une voie d'intensification durable importante des systèmes de production agricole en régions chaudes, comme elle l'a été historiquement pour nos agricultures du Nord. Mais les contextes ont beaucoup évolué et il faut, bien sûr, revisiter ce débat récurrent.Les fortes évolutions observées récemment, sous l'effet notamment de la croissance démographique qui reste forte au Sud et de l'urbanisation qui s'y accélère aussi, invitent à reconsidérer les caractéristiques des milieux auxquels on s'intéresse et les perspectives de développement durable qui s'y présentent; il faut en effet tenir compte dans nos analyses et propositions de la densité agricole et humaine, de l'état des ressources naturelles, de l'importance des troupeaux, des marchés, de l'accès aux intrants, etc. En outre, les situations agraires sont souvent bien différentes dans les régions chaudes des pays en développement du Sud et dans les agricultures des pays industrialisés du Nord pour de nombreuses raisons historiques, économiques et politiques.Aussi, les relations entre l'agriculture et l'élevage qui en résultent peuvent apparaître comme des effets de ces différents facteurs et de ces évolutions, mais aussi comme des indicateurs qui permettent (parmi d'autres) de caractériser ces diverses situations.Sans vouloir être exhaustif, nous pouvons tenter de rappeler certaines caractéristiques fondamentales de ces relations agriculture-élevage.

\section{Interactions et synergies positives}

Traditionnellement, on considère que cette association permet des interactions, des synergies positives, notamment en favorisant différents flux favorables entre les activités de culture et d'élevage au sein d'une unité de production donnée (ou à d'autres niveaux : terroir, petite région, etc.).

De quels flux s'agit-il [4] ?

- - de l'énergie développée par les animaux de trait et de bât en faveur des cultures, par la culture attelée (divers travaux culturaux : labours, semis, sarclages, buttage, etc.) et par le transport des biens (intrants agricoles, fumier, fourrages, récoltes, etc.) ; 
- - des aliments destinés aux animaux et provenant en partie du système de culture (fourrages, résidus de récolte, jachères, etc.) ;

- - des éléments fertilisants produits par les animaux (la "fumure animale » : déjections, fumier) et permettant de fertiliser les champs.

On a coutume de dire qu'il s'agit-là des trois piliers biotechniques de l'association de l'agriculture et de l'élevage ; il faut bien sûr y ajouter les nombreux flux économiques : les revenus des ventes des cultures permettant par exemple d'acquérir des animaux ou la vente d'animaux finançant le matériel agricole..., nous y reviendrons.

II est intéressant de dépasser ces éléments de base grâce à d'autres considérations complémentaires.

\section{Intensification raisonnée des productions agricoles (cultures et élevage)}

L'intégration de l'agriculture et de l'élevage permet une intensification simultanée et synergique des productions végétales et animales répondant aux objectifs d'accroissement des productions, des revenus des ménages agricoles et d'usage plus efficace des ressources. Ces synergies, nous l'avons déjà évoqué, concernent les flux de matières, les cycles de nutriments, le fonctionnement biophysique des sols, la valorisation du travail, la gestion des facteurs de production (équipements et intrants). Chez les plus pauvres, cette forme d'intensification relative présente l'intérêt majeur d'une réelle autonomie (peu d'intrants à acquérir à l'extérieur).

\section{Diversification, diminution des risques économiques}

La promotion de l'AAE peut se concevoir par différentes voies que l'on peut schématiser en disant qu'elle peut se développer à partir de l'agriculture, voie sans doute la plus classique, ou à partir de l'élevage. Elle se traduit en général par une diversification des productions de l'exploitation agricole, ce qui est sans doute une façon de la sécuriser économiquement par rapport à l'aléa climatique ou aux fluctuations des prix des produits agricoles. Le petit élevage peut jouer à ce titre un rôle économique important (espèces à cycle court notamment). Les grands herbivores, équidés, bovins, bubalins, ont aussi une fonction multiple en apportant souvent une source d'énergie essentielle mais aussi du fumier, le transport attelé permettant justement de gérer les flux de matière organique (fourrages, fumier...).

\section{Optimisation et réduction de la pénibilité du travail}

L'introduction d'animaux pour le travail est une forme importante de l'AAE [5] ; elle se traduit en général par une nouvelle combinaison du travail humain et animal et par une forte réduction de la pénibilité du travail manuel (c'est-à-dire des personnes); à lui seul, ce dernier point permettrait souvent de justifier le recours au travail animal ; il permet en effet une meilleure efficacité et qualité du travail (labour ou buttage, par exemple), une plus grande rapidité d'intervention (semis) et un meilleur " rendement » (transport attelé par exemple). Globalement, c'est la productivité du travail humain qui peut en être fortement améliorée. 


\section{Amélioration de l'économie familiale, de la trésorerie et de la sécurité alimentaire}

Les animaux permettent souvent de diversifier dans le temps les revenus de l'exploitation; de plus, c'est parfois la trésorerie au quotidien qui peut être notablement améliorée avec des produits animaux tels que les œufs, les poulets, le lait, les petits ruminants, etc. L'autoconsommation de certains produits animaux (lait, petit élevage) contribue qualitativement (protéines à haute valeur biologique) à l'alimentation et à la sécurité alimentaire de la famille.

Les produits animaux permettent de sécuriser l'économie et de réguler la trésorerie. La vente du coton s'effectue après la récolte, une fois par an, celle de nombreux produits animaux peut s'étaler dans le temps et parfois se décaler dans l'année (animaux sur pied notamment). C'est en raison de cette souplesse que l'on peut observer autant de labilité de ces animaux dans les exploitations: accumulation et déstockage animal sont des facilités offertes aux familles pour réguler leurs besoins monétaires.

L'organisation sociale de ces productions animales peut être complexe, ce qui ne facilite pas la prise de décision, ni le conseil car " il ne faut pas se tromper d'interlocuteur " [4]. On peut y voir une cause du plafonnement des progrès de productivité des animaux dans ces systèmes.

Mais la multifonctionnalité des animaux est un atout important : la vache de trait, par exemple, peut assurer des travaux légers (avec certains avantages sur les mâles [4]), produire du lait et des veaux, ainsi que du fumier : il s'agit d'une forme accomplie de l'intégration de l'élevage et de l'agriculture.

\section{Valorisation des ressources du terroir}

L'introduction des animaux dans l'exploitation agricole permet une meilleure valorisation d'un ensemble de production ou de coproduits tels que: les résidus de récolte, les sous-produits domestiques, artisanaux et agro-industriels... Les herbivores jouent un rôle spécifique dans cette valorisation car ils sont capables d'utiliser des fourrages pauvres provenant des jachères, des parcours et des récoltes (résidus et sous-produits).

Globalement on peut dire que l'association de l'agriculture et de l'élevage contribue à l'allègement de la pauvreté et à la durabilité des systèmes de production agricoles dans les pays en développement.

\section{Energie et fumure animales}

Revenons sur deux aspects importants de l'AAE que sont l'entretien de la fertilité des sols et l'utilisation de l'énergie animale qui illustrent notamment des différences essentielles entre les contextes Nord et Sud.

L'entretien de la fertilité des sols est au cœur de la problématique de la durabilité des systèmes mixtes agriculture-élevage.

Dans le système " polyculture-élevage ", la diversification et la rotation des cultures et l'introduction de cultures fourragères peuvent favoriser grandement l'entretien de la fertilité des sols, mais l'utilisation raisonnée de la fumure animale peut aussi y contribuer significativement. De nouveaux systèmes de culture sous couverture végétale (vivante ou morte), les SCV, vont aussi dans le sens d'une gestion agro-écologique durable des ressources; I'animal peut y trouver sa place, mais cela 
complexifie le système de production et pose de nouveaux problèmes, tant pour la mécanisation attelée que pour le système d'alimentation et la conduite des animaux de trait et des troupeaux. La valorisation de la fumure organique animale pour l'entretien de la fertilité des champs cultivés fait l'objet de pratiques agricoles très diverses [6].

Les aspects favorables de l'apport de matière organique (fumier, compost) aux sols tropicaux sont divers :

- - apport d'éléments fertilisants ;

- - amélioration de la capacité de fixation et d'échange d'éléments nutritifs ;

- - amélioration des caractéristiques physiques des sols et de la résilience des sols fragiles par amélioration de leur structure et augmentation de leur stabilité et de leur résistance ;

- - stimulation de la vie biologique du sol (microflore et microfaune) ;

- - amélioration des capacités de rétention de l'eau.

Avec les herbivores, on peut observer un transfert de fertilité (redistribution dans le temps et dans l'espace des éléments fertilisants) permis par ces animaux qui sont susceptibles de pâturer sur certaines unités de ressources (parcours, forêts, jachères, etc.) pour déposer leurs déjections sur les parcelles cultivées: il existe, à ce titre, en Afrique subsaharienne, des pratiques de parcage, des contrats de fumure, etc. qui témoignent de l'intérêt que portent les agriculteurs à ces transferts. $\mathrm{Ce}$ sont certes des solutions modestes et limitées, mais " autonomes " et peu coûteuses qui permettent aux paysans pauvres de valoriser leurs maigres ressources et notamment les déjections animales.

Paradoxalement, ces éléments fertilisants qui apparaissent comme une richesse à bien valoriser dans la majorité des systèmes de production des pays en développement peuvent devenir, en raison justement de leur excès d'abondance, une grave source de pollution dans les systèmes intensifs des pays industrialisés. On peut aussi se demander pourquoi la logique de l'AAE ne s'est pas plus franchement imposée dans certains systèmes économiques; le rôle des politiques publiques (manque de garanties foncières, engrais subventionnés, etc.) en la matière n'est sans doute pas négligeable.

L'utilisation de l'énergie animale dans les systèmes de production agricole des pays en

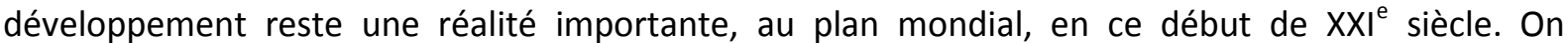
considère classiquement ( $F A O)$ que plusieurs centaines de millions d'animaux (bovins bubalins, équidés, camélidés, etc.) participent à cette fourniture d'énergie dans l'agriculture, à travers le travail du sol et les transports.

En Afrique subsaharienne, en particulier, l'équipement des exploitations agricoles présente de très grandes disparités interrégionales mais aussi entre les exploitations d'une même région. La part de l'énergie humaine dans les systèmes agricoles est encore majoritaire, ce qui justifie amplement de continuer d'œuvrer pour une meilleure intégration de l'agriculture et de l'élevage à travers les animaux pour le travail.

Dans les pays industrialisés, souvent marqués par une très grande spécialisation des systèmes de production (tant en élevage qu'en culture), on peut se demander si des systèmes mixtes faisant 
appel à l'AAE, ainsi qu'à l'agroforesterie et à une approche plus écologique de la production agricole ne permettraient pas de réduire certaines externalités très coûteuses pour la collectivité (érosion, ruissellement, inondations, pollutions, feux, etc.).

\section{L'association de l'agriculture et de l'élevage : un modèle durable ?}

Le modèle classique d'AAE est appelé à évoluer et ses limites sont parfois évidentes [7]. On observe ainsi parfois sa régression en raison de tendances de nature différente :

- - c'est parfois l'atomisation des exploitations agricoles familiales et la dégradation des ressources naturelles qui remettent en cause la présence de gros animaux dans les unités de production (hauts plateaux éthiopiens par exemple) : ces évolutions sont les conséquences d'une forte augmentation démographique, sans solution de travail extérieur et elles se traduisent parfois par la régression d'un système complexe polyculture-élevage et par la paupérisation dramatique des familles rurales concernées ;

- - l'évolution des systèmes agricoles conduit parfois à la spécialisation, ce qui remet aussi en cause l'AAE ; ce sont alors les forces du marché, les nouvelles technologies plus performantes et l'évolution économique qui poussent à l'agrandissement et à la spécialisation des unités de production.

Il en résulte parfois des problèmes environnementaux, comme nous l'observons aussi dans nos pays " développés ". Pour les pays en développement les plus pauvres, l'AAE apparaît bien comme une solution pertinente pour améliorer la durabilité des systèmes de production familiaux, alléger la pauvreté des petits producteurs et réduire leur dépendance économique.

\section{Références}

1 DUFUMIER M. Un agronome dans son siècle. Actualité de René Dumont. Karthala, Paris : INAPG, 2002.

2 LHOSTE P. Le riz sans la viande est triste. René Dumont et l'élevage en Afrique tropicale 2002 : 8794.

3 MAZOYER M, ROUDART L. Histoire des agricultures du Monde. Du néolithique à la crise contemporaine. Paris : Le Seuil, 1997.

4 LHOSTE P. L'association agriculture-élevage. Evolution du système agropastoral au Siné-Saloum (Sénégal). Paris : INAPG, Cirad, 1986.

5 PEARSON RA, LHOSTE P. Working animals in agriculture and transport. A collection of some current research and development observations. Wageningen Academic Publishers, The Netherlands, 2003 ; EAAP Technical series $\mathrm{N}^{\circ} 6$.

6 LANDAIS E, LHOSTE P, GUERIN H. Les systèmes de gestion de la fumure animale et leur insertion dans les relations entre l'élevage et l'agriculture. Cahiers Agricultures $1993 ; 2: 9-25$.

7 LANDAIS E, LHOSTE P. L'association agriculture-élevage en Afrique intertropicale : un mythe techniciste confronté aux réalités du terrain. Cahiers des Sciences Humaines 1990 ; 26(1-2) : 217-35. 